\title{
The Diagnosis of Unilateral Diaphragmatic Paralysis Using M-mode: A Case Report from Palestine
}

\author{
Mohammed Khatib, Muayad Salman, Sami Smerat and Marah Abu Muhsen
}

\section{ABSTRACT}

Background: Diaphragmatic paralysis is one the causes of dyspnoea and difficulty in breathing. There are many causes of diaphragmatic paralysis, especially trauma and iatrogenic injury of phrenic nerve. Usually, diaphragmatic paralysis diagnosis depends on fluoroscopic examination of diaphragmatic displacement during sharp sniffs. However, due to disadvantages of fluoroscopic examination, the trend for less radiation examination by using ultrasound is increasing especially using M-mode for detection of diaphragmatic motion during respiration.

Case: A new-born baby boy with uneventful pregnancy and normal delivery with good general condition, developed severe respiratory distress at $3^{\text {rd }}$ day of life and was diagnosed to have bilateral pneumothorax. Bilateral chest tubes were inserted with improvement of his condition. The baby then started to develop desaturation with serial chest $x$-rays done and showed elevated left hemi-diaphragm. Suspicion toward left hemi-diaphragm paralysis increased and due to his general condition, fluoroscopic examination was difficult. So that, Ultrasound with M-mode was used with diagnosis of the patient with left hemi-diaphragmatic paralysis.

Conclusion: Ultrasound and the using of M-mode in detection of diaphragmatic motion is one of the most important and applicable diagnostic procedure for diagnosis of diaphragmatic weakness and paralysis.

Keywords: Motion mode, neonatal intensive care unit, ultrasound.

Submitted : September 08, 2021

Published : November 03, 2021

ISSN: 2593-8339

DOI: $10.24018 /$ ejmed.2021.3.6.1055

M. Khatib

Istishari Arab Hospital, Palestine. (e-mail: m_taisir1993@yahoo.com) M. Salmañ*

Al-Makassed Hospital, Palestine.

(e-mail: muyad.salman@iah.ps)

S. Smerat

Istishari Arab Hospital, Palestine. (e-mail: ssmerat@paluniv.edu.ps) M. Abu mhusen

Istishari Arab Hospital, Palestine. (e-mail: marahabumuhsen@iah.ps)

*Corresponding Author

\section{INTRODUCTION}

Dysfunction of the diaphragm is an important, underdiagnosed cause of breathlessness. In unilateral diaphragm weakness, the presentation can be asymptomatic or with difficulty in breathing on activity, and it is frequently discovered incidentally [1]. Diaphragmatic dysfunction can range from a partial loss of ability to generate pressure (weakness) to a complete loss of diaphragmatic function (paralysis). Diaphragmatic weakness or paralysis can affect one or both hemi-diaphragms and can occur as a result of metabolic or inflammatory disorders, trauma, or surgery, during mechanical ventilation, as well as those with mediastinal masses, myopathies, neuropathies, or diseases causing lung hyperinflation [2]. In clinical practice, diagnosis of diaphragmatic paralysis is usually and traditionally based on the fluoroscopic examination of diaphragmatic displacement during sharp sniffs [3]. In this procedure, sniffing causes forceful contraction and caudal motion of the intact half of the diaphragm, the fall in pleural pressure and the rise in abdominal pressure tend to displace the paralyzed half of the diaphragm cranially [3]. Despite fluoroscopic wide use in diagnosis of diaphragmatic paralysis, it has many disadvantages limit it use. Despite its widespread use in the diagnosis of diaphragmatic paralysis, fluoroscopic imaging has a number of drawbacks that limit its utility. Fluoroscopy necessitates patient cooperation and the ability of the patient to remain upright. It also involves a significant radiation dose on the patient and produces difficult-to-replicate results [4]. $\mathrm{U} / \mathrm{S}$ is a technique still insufficiently used in respiratory medicine. However, many advantages can be seen in using ultrasound over fluoroscopic examinations: it is easy to use, there is no irradiation, and it can be used at the bedside, particularly when the patient is immobile or in intensive care. Ultrasound allows morphological and dynamic study of each hemi-diaphragm, also it provides important information on the thoracic and sub-diaphragmatic environment [5]. Ultrasonography in B-mode or M-mode has proven useful in assessing and evaluating diaphragmatic function [6]. M mode ultrasonography has been reported as a useful technique that is not yet in general use worldwide, having received some attention in the adult population, but primarily being a modality used in the pediatric population [7]. M-mode sonography records the successive positions of a structure versus time, allowing the quantification of normal or abnormal diaphragmatic motion [8]. The normal diaphragm contracts and moves caudally toward the transducer during inspiration. This is represented by an upward movement of 
the M-US tracing. The diaphragm moves cephalad, away from the transducer, during expiration. as a result of which the M-US tracing has a downward inflexion The M-US tracing examines two parameters: motion direction and excursion amplitude. Diaphragmatic motion can be normal, reduced, absent, or paradoxical. If the diaphragmatic motion in inspiration is toward the transducer and the excursion is greater than $4 \mathrm{~mm}$, this is considered normal Moreover, the excursion difference between the hemi-diaphragms is less than $50 \%$. Decreased excursion is considered when the amplitude is less than $4 \mathrm{~mm}$ and the amplitude difference between the hemi-diaphragms is greater than $50 \%$ (Fig. 1). When the tracing shows a flat line, absent motion is considered. When diaphragmatic motion is directed away from the transducer during inspiration, paradoxical motion is considered. Diaphragmatic paralysis is indicated by lack of motion and paradoxical motion [9] (Fig. 1).

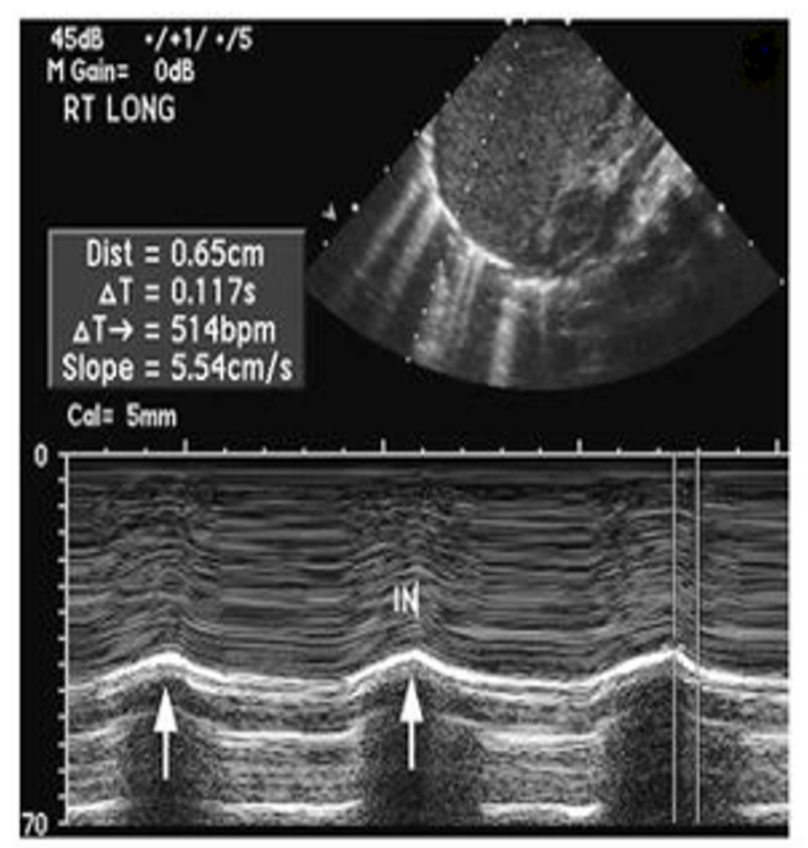

Fig. 1. M-mode sonography of the normal hemi-diaphragm with abovebaseline inspiratory peak (arrows).

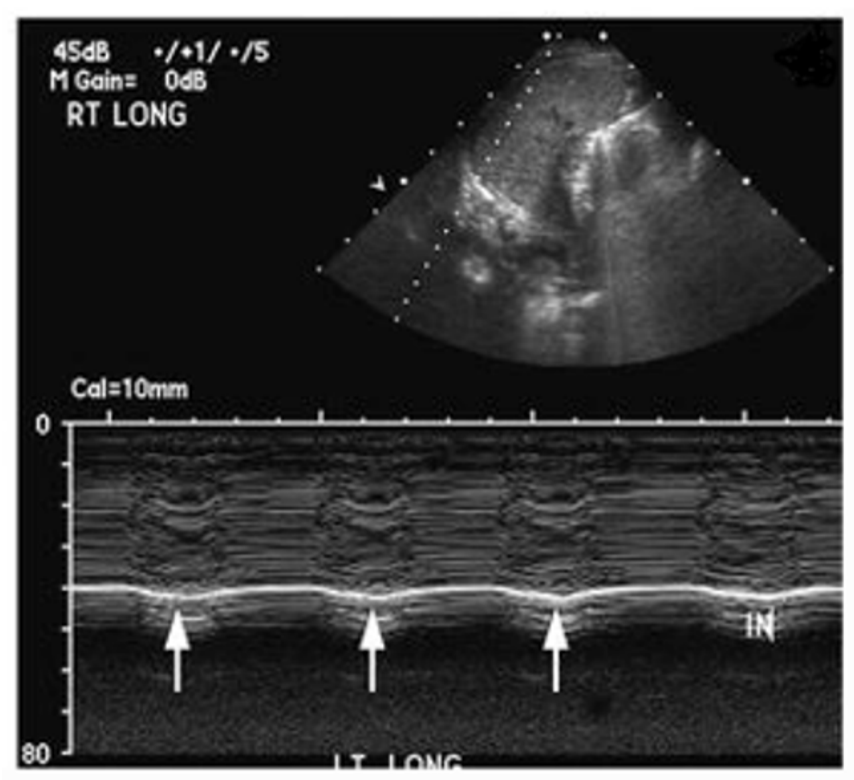

Fig. 2. M-mode sonography of the paralyzed hemi-diaphragm characterized by a small paradoxical inspiratory peak below the baseline (arrows).

\section{CASE REPORT}

In this case we are going to talk about a newborn male baby, who was born as a product of normal spontaneous vaginal delivery at full term good health state, good respiratory status, and normal birth weight, with no complication during pregnancy or delivery. His health status was good until $3^{\text {rd }}$ day of delivery, when the patient suddenly developed deterioration in his general health condition, which was representing as decreased $\mathrm{O}_{2}$ saturation below 90, difficulty in breathing, respiratory distress, and bilateral lung fields decreased air-entry on auscultation. Chest x-ray was done and shown bilateral tension pneumothorax. So that, patient was urgently intubated with insertion of bilateral chest tubes. After that patient was admitted to NICU and he started on mechanical ventilation with bilateral chest tubes for his bilateral pneumothorax. Patient was followed in NICU with observation of his vital signs, daily examination, and following chest $\mathrm{x}$-ray for his bilateral pneumothorax. His condition was improving, so that after 5 days patient was extubated and he was started on nasal $\mathrm{O}_{2}$ therapy with removal of right-side chest tube due to more than 24 hours non-functioning state, in addition to clamping of left sided chest tube. After 24 hours of left sided chest tube clamping and chest x-ray showed that there was no left sided pneumothorax, left sided chest tube was removed and patient was kept under observation and follow-up. After that, patient was started on antibiotics due to elevated inflammatory markers and patient was treated as a case of neonatal sepsis. His respiratory condition was not improved well, and he continued to develop desaturation and continued nasal $\mathrm{O}_{2}$ therapy with the aid of non-invasive mechanical ventilation. His chest $\mathrm{x}$-ray showed chest infection and he was treated by antibiosis due to his chest infection. Respiratory status of the patient was the same with no improvement in his condition and his serial blood gases shown mixed respiratory and metabolic acidosis. Despite his heavy management with antibiotics, nebulizers, and non-invasive mechanical ventilation patient condition did not improve. With the follow of patient's serial chest $x$-rays with pediatric radiologist there was an elevation of patient's left hemi-diaphragm over right one. This finding raises the suspicious of left sided hemidiaphragm paralysis due to left sided phrenic nerve injury after chest tube insertion on left side. The decision was to do traditional fluoroscopic examination for diagnosis of left sided hemi-diaphragmatic paralysis. But, due to patient's condition and the difficulty for patient transportation to radiology department, decision was to depend on ultrasound for diagnosis of diaphragmatic paralysis. On evaluation of right hemi-diaphragm, there was good movement of the hemi-diaphragm toward probe during inspiration, and the $\mathrm{M}$ mode showed that there was normal movement of the right hemi-diaphragm (Fig. 3). On the other side, left hemidiaphragm showed no movement during respiration with $\mathrm{M}$ mode showed no movement of the diaphragm line, and this suggestive of left hemi-diaphragm paralysis (Fig. 4). Patient was diagnosed as a case of left sided hemi-diaphragm paralysis after iatrogenic left phrenic nerve chest tube trauma by using M-mode ultrasound with no need of fluoroscopy. After that, patient treated conservatively in NICU with improvement in his general condition and respiratory issue. 


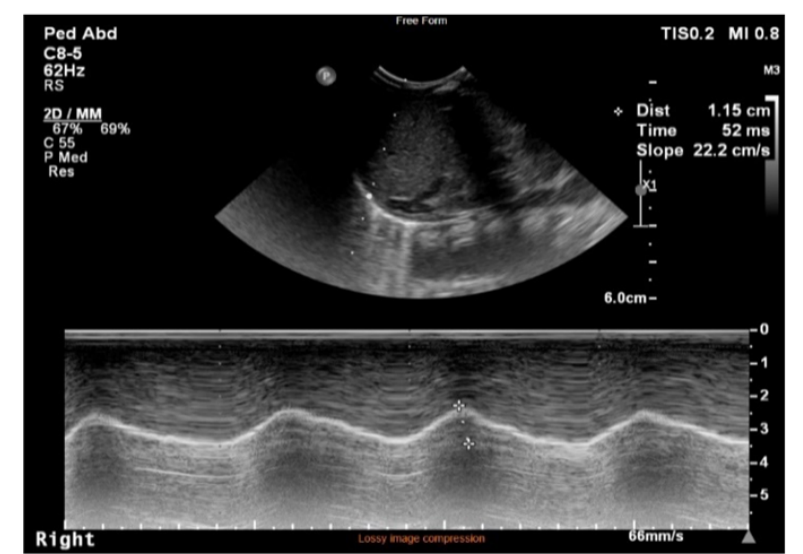

Fig. 3. M-mode of right hemi-diaphragm with normal movement during respiration.

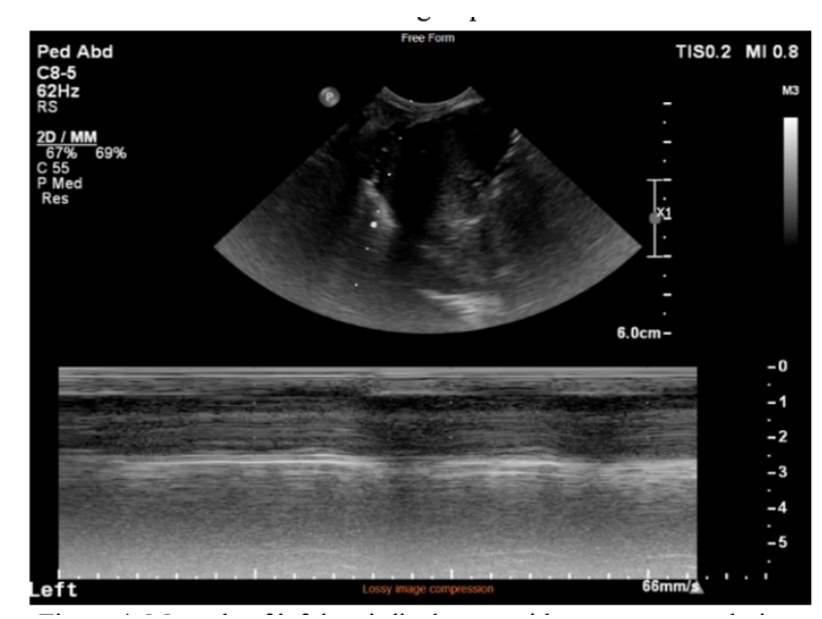

Fig. 4. M-mode of left hemi-diaphragm with no movement during respiration.

\section{DISCUSSION}

The diaphragm is the primary breathing muscle, and its contraction is essential for respiration, so that any diaphragmatic dysfunction will be associated with difficulty in breathing or other respiratory symptoms [10]. This mean, it is important for good diagnosis and proper treatment of diaphragmatic paralysis, in addition to identifying the most common causes of diaphragmatic injuries, particularly in children.

Diaphragmatic paralysis is a rare but significant cause of respiratory distress in infants. in the first years of life, the main cause of diaphragmatic paralysis or weakness is phrenic nerve injury after iatrogenic trauma such as surgeries, or obstetrical trauma [11]. As shown in our case, the cause of left sided diaphragmatic injury was phrenic nerve injury after chest tube insertion. so that, although it is rare, but diaphragmatic paralysis should be considered after iatrogenic trauma associated with respiratory distress. diaphragmatic paralysis is usually suspected with chest $\mathrm{x}$-ray which is showing abnormal hemi-diaphragm elevation [11]. In above case, the suspicion for diaphragmatic paralysis was due elevated hemi-diaphragm on serial chest with respiratory distress. fluoroscopy was considered the gold standard for diaphragmatic paralysis diagnosis, but it has gradually been replaced by ultrasound, which can be done at the bedside especially in critically it patient [11].
Fluoroscopy has traditionally been the imaging modality of choice for suspected paralyzed diaphragms (the default gold standard). on the other hand, identified a number of limitations in the use of fluoroscopy in the diagnosis of diaphragmatic paralysis. it necessitates patient cooperation as well as the patient's ability to remain upright, also it involves a high radiation dose to patient, and in addition, its results are difficult to reproduce [4]. These limitations and others show us the limitations for use of fluoroscopy in diagnosis of diaphragmatic paralysis especially in pediatric age group. $\mathrm{M}$ mode ultrasonography is a relatively simple and accurate test for diagnosing paralysis of the diaphragm, because it allows better evaluation of movement and accurate measurements in moving structures [7]. There are many studies worldwide that studies the use of $\mathrm{u} / \mathrm{s}$ and m-mode in detection of diaphragmatic movements and its accuracy in diagnosis of diaphragmatic motion abnormalities. All of these studies show that m-mode is the preferred mode for assessing diaphragmatic motion and is an accurate test for diaphragmatic paralysis diagnosis especially in critically ill patient and pediatric patients in addition it is useful in the follow-up of these patients [6], [7], [9], [12], [13].

\section{CONCLUSION}

$\mathrm{U} / \mathrm{S}$ and the using of M-mode in detection of diaphragmatic motion is one of the most important and applicable diagnostic procedure for detection of diaphragmatic movements, which helps in diagnosis of diaphragmatic weakness and paralysis especially in pediatric and critically ill patients.

\section{REFERENCES}

[1] Caleffi-Pereira M, Pletsch-Assunção R, Cardenas LZ, Santana PV, Ferreira JG, Iamonti VC, Caruso P, Fernandez A, de Carvalho CR, Albuquerque AL. Unilateral diaphragm paralysis: a dysfunction restricted not just to one hemidiaphragm. BMC pulmonary medicine. 2018 Dec;18(1):1-9.

[2] McCool FD, Tzelepis GE. Dysfunction of the diaphragm. New England Journal of Medicine. 2012 Mar 8;366(10):932-42.

[3] Scillia P, Cappello M, De Troyer A. Determinants of diaphragm motion in unilateral diaphragmatic paralysis. Journal of applied physiology. 2004 Jan;96(1):96-100.

[4] Houston JG, Angus RM, Cowan MD, McMillan NC, Thomson NC. Ultrasound assessment of normal hemidiaphragmatic movement: relation to inspiratory volume. Thorax. 1994 May 1;49(5):500-3. [5] P. Richard, "Exploration du diaphragme: l'échographie est incontournable," Revue des Maladies Respiratoires 34(6): 645-660, 2017.

[6] Boussuges A, Brégeon F, Blanc P, Gil JM, Poirette L. Characteristics of the paralysed diaphragm studied by M-mode ultrasonography. Clinical physiology and functional imaging. 2019 Mar;39(2):143-9.

[7] Lloyd T, Tang YM, Benson MD, King S. Diaphragmatic paralysis: the use of $\mathrm{M}$ mode ultrasound for diagnosis in adults. Spinal cord. 2006 Aug;44(8):505-8..

[8] Gerscovich EO, Cronan M, McGahan JP, Jain K, Jones CD, McDonald C. Ultrasonographic evaluation of diaphragmatic motion. Journal of ultrasound in medicine. 2001 Jun;20(6):597-604. [9] M. Epelman, O. M. Navarro, A. Daneman and S. F. Miller, "M-mode sonography of diaphragmatic motion: description of technique and experience in 278 pediatric patients," Pediatric Radiology 35(7):661-7, 2005.

[9] Epelman M, Navarro OM, Daneman A, Miller SF. M-mode sonography of diaphragmatic motion: description of technique and experience in 278 pediatric patients. Pediatric radiology. 2005 Jul;35(7):661-7.

[10 J. Ricoy J, Rodríguez-Núñez N, Álvarez-Dobaño JM, Toubes ME, Riveiro V, Valdés L. Diaphragmatic dysfunction. Pulmonology. 2019 Jul 1;25(4):223-35. 
[11] Gerard-Castaing N, Perrin T, Ohlmann C, Mainguy C, Coutier L, Buchs C, Reix P. Diaphragmatic paralysis in young children: A literature review. Pediatric pulmonology. 2019 Sep;54(9):1367-73.

[12] Hamadah HK, Kabbani MS, Elbarbary M, Hijazi O, Shaath G, Ismail S, Qadi AM, AlTaweel H, Jijeh A. Ultrasound for diaphragmatic dysfunction in postoperative cardiac children. Cardiology in the Young. 2017 Mar;27(3):452-8.

[13] M. Riccabona, E. Sorantin and E. Ring, "Application of M-mode sonography to functional evaluation in pediatric patients," European Radiology 8(8): 1457-1461, 1998.

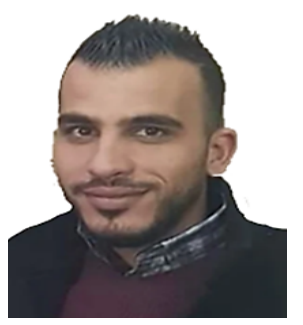

Sami Smerat was born in the city of Hebron, Palestine. He received his B.A. degree in Radiography from Palestine Ahliya university in Bethlehem, Palestine in 2016. Moreover, he was a Research assistant (RA) and Lecturer in Palestine Ahliya university for four years. He has worked as a Radiologic technologist in various private clinics and is currently working at Istishari Arab Hospital. He is a proactive researcher and has published research regarding diagnostic imaging and physiotherapy. His areas of interest are physiotherapy and ultrasound.

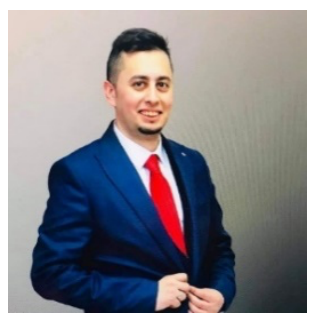

Mohammed Khatib was born in the town of Attil in Palestine. He earned B.A. degree in Medicine from Najah National University Nablus in 2017. After that, he finished the Internship year in governmental hospitals of Palestinian Ministry of Health in 2018. On June 2020, he joined medical diagnostic radiology residency program at Department of Diagnostic Radiology at Istihsari Arab Hospital - Ramallah, Palestine.

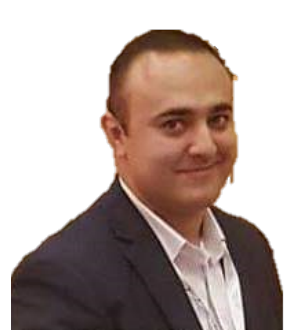

Muayad Salman was born in Kuwait. He is a consultant radiologist with up to 10 years of experience. $\mathrm{He}$ is currently a radiology consultant at Al-Makasad Hospital and Istihari Hospital. Dr. Salman is also an assistant professor at Al-Quds University- Jerusalem. He graduated from the Faculty of Medicine from Quaid-e-Azam Medical College- Pakistan in 2006, then he continued his specialized training in Diagnostic Radiology at AlMakssed Hospital in 2008. He completed his fellowship of pediatric radiology at Sickkids Hospital in Canada. Furthermore, he participated as a board examiner for Palestinian Diagnostic Radiology. In addition, Dr. Salman has participated in many radiologic conferences, the most recent being in Germany in the year 2018. He has published research concerning pediatric Meckel diverticulum and juvenile spondylarthritis.

Marah Abu Muhsen was born in the city of Nablus in Palestine. She acquired her B.A. degree in radiologic technology from the Arab American University in the year 2015. Since then, she has been proactive in the field of radiography: she earned Philips Healthcare certifications for intensive training in MRI and CT in the year of 2016. She currently works in the radiology department of Istishari Arab Hospital as a radiologic technologist and is a member of the quality control (QC) staff. She acquired the award of "best radiologic technologist" in the radiology department of Istishari Arab Hospital for many consecutive years. Her active participation as a quality control officer inspired her to spread awareness about breast cancer. Marah participated in conferences that aim to raise breast cancer awareness and scrutinize quality control in the year 2017 and 2020 that were held by Al-Ahliya University and Medical Imaging Association respectively. 